\title{
ORTS- UND SACHREGISTER
}

Aachen/Aix-la-Chapelle 28

Académie royale des sciences et belleslettres de Berlin 219, 221, 224-227, 229, 235-237, 239

Adelsheim 44

Afrika/Afrique 233

Aisch 107, 115

Allgemeines Preußisches Landrecht 7883

Altheim 119f

Altona 126, 131

Amerika/Amérique 134

Amsterdam 38, 44, 127-131, 142

Anglikanische Kirche/Église anglicane 37

Annweiler 28, 31

Ansbach-Bayreuth 44

Antillen, französische/Antilles françaises 128f., 133

Antwerpen/Anvers 28, 30

Archenweyer 25

Artois 30,41

Asien/Asie 233

Aufklärung/Lumières $12,171,178,185$, 187-192, 211-230, 238

Augsburgische Konfession/confession d'Augsbourg 8,52

Aunis 7

Babylon/Babylone 228

Baiersdorf 107, 114f

Baltikum/Baltique 32, 131

Barberot 31

Bartholomäusnacht/Saint-Barthélemy, nuit de la (1572) 37

Basel/Bâle 41, 86

Battin, Uckermark/Marche Ukraine 155169

Bayern/Bavière 19,21

Bayreuth 44, 115, 234

Bergel 119, 122

Bergzabern 29

Berlin 9, 11-13, 41, 58, 60, 63, 85-106, $147,152,155-169,171,173 f$., 176 , 179-181, 187, 193-208, 211-230, 234 239
Besançon 234

Béziers 222

Billigheim 25, 31

Böckelheim 18

Bockenheim 38, 40, 43f.

Bonneville, Picardie 40

Bordeaux 127-129

Bourg 89, 106

Brandenburg, Havel/Brandebourg, Havel 156

Brandenburg-Ansbach/BrandebourgAnsbach 116

Brandenburg-Bayreuth/Brandebourg-

Bayreuth 11, 107-124

Brandenburg-Preußen/BrandebourgPrusse 9, 11-13, 25, 38, 43f., 48, 53, $56,58,64,68,71-83,85 f$., 90-92, 97, 99 , 10lf., 106f., 113, 116, 118, 126, 130, 134, 139-169, 171f., 175f., 178, $182,184,186 f$., 190-195, 201, 204 206, 211f., 216-220, 228, 230, 231-240

Brasilien/Brésil 128, 133

Braunsberg, Ruppin 156

Bremen/Brême 131

Bretagne 130

Brüssel/Bruxelles 30

Burgbernheim 119, 122

Calais 27

Calwer Zeughandlungskompagnie 21

Catéchisme de Superville 200

Cevennen/Cévennes 42, 97

Champagne 239

Chancellerie française

- in Hessen-Kassel/en Hesse-Cassel 73, 75

Charenton 211

Charleston 99

Clairac, Lot 130

Clingen 25

Collège françois, Hochschule/école supérieure 163, 214, 222, 229

Compagnie de Caracas 130

Compagnie du Consistoire 196, 199-202, 203, 208 
Concessions et Privileges $72-74$

Confessio Augustana invariata 8, 52

Connewitz 65

Corpus Evangelicorum 55

Courthézon 94, 98

Dänemark/Danemark 231

Dauphiné 7, 40, 42

Dessau 174

Deutsche reformierte Kirche/Église allemande réformée $38,42,162,201 \mathrm{f}$

Deutschland/Allemagne 9, 12f., 40, 46, $130,134,139,149,153,173,188 \mathrm{f}$., 224, 231f., 234, 237

Dezemberdekret von Hessen-Kassel 77

Dictionnaire de l'Académie française $166 f$.

Dorotheenstadt 87

Dreißigjähriger Krieg/guerre de Trente Ans $8,18,20,25,27 \mathrm{f}$., 48, 53, 55, 130 , 143,159

Dresden/Dresde $52,58,62,65,68 \mathrm{f}$

Düsseldorf 41

Edikt von Fontainebleau/£dit de Fontainebleau (1685) 7f., 39, 53, 72f., $86,125,135,173-175,195,214 f$., 222 , 226, 231

Edikt von Nantes/édit de Nantes (1598) $7,24,61,72,135,171,194 f$., 214, 222 , 226

Edikt von Potsdam, »Potsdamer Edikt $U$ édit de Potsdam (1685) 11, 33, 58, 72, 76-78, 80f., 86, 113, 142-146, 155f., $172,193,195,197,211,235$

Edingen 28

Eilenburg 55

Eintrachtskirche in Mannheim 21

Elbe 126, 128

Elsaß/Alsace 7

Encyclopédie ou dictionnaire raisonné des sciences, des arts et des métiers, hg. von/éditée par d'Alembert et Diderot 181f., 225

England/Angleterre 8-10, 19, 28, 36, 55f., $76 f$., 82, 85f., 89, 126, 128, 212 , $215,223,228,231 \mathrm{f}$.

- Kolonien/colonies 8

Erlangen, Christian-Erlang 44, 62, $107 f$., $115,117 \mathrm{f} ., 120 \mathrm{f}$

Erlenbach 25
Europa/Europe 8f., 39, 60, 85, 98, 102, $125,128,130 \mathrm{f} ., 133-135,174,185$, $189,216,219,226,237-239$

exercitium religionis privatim 62

Fahrenwalde 156

Fiume, heute/de nos jours Rijeka 128

Flämische Kirche/Église flamande 37, 42

Flandern/Flandre 25, 27

Flüchtlings- und Ansiedlungskommission, landgräfliche Kommission in HessenKassel 73

Fontainebleau siehe/voir Edikt von Fontainebleau/édit de Fontainebleau

Francheval 32

Franchimont 18

Franken/Franconie 9

Frankenthal 18, 27f., 30f.

Frankfurt am Main/Francfort-sur-le-Main $11,18,27,29,32,35-49,86,142,229$, 232

Frankfurt an der Oder/Francfort-surl'Oder 156f., 202, 206, 213, 235

Frankreich/France 7, 12, 25, 31, 38f., 42, $47,51,54,56,60,63 f ., 67,71 \mathrm{f} ., 76 f$., $80,85 f$., 125, 127f., 132-135, 139-144, 148f., 151, 153, 166-168, 172, 178, $183-187,189,191,194,213,215,220$ 224, 226-228, 231f., 234, 236-239

Französische Kolonie in BrandenburgPreußen/Colonie française au Brandebourg-Prusse 146f., 149-152, $156,159,171-176,178,180$ f., 184 , 186, 191f., 208, 235

Französische Kommission, früher Commission française, in BrandenburgPreußen/Französische Kommission, jadis Commission française, au Brandebourg-Prusse 78

Französische Nationalversammlung/Assemblée nationale 146, 148f., 153

Französische reformierte Kirche/Église française reformé $37 \mathrm{f}$., $40,42 \mathrm{f}$., 49 , $87,98,101,147,150,156,162 \mathrm{f} ., 179 \mathrm{f}$., 195f., 198

Französische Revolution/Révolution française 153,191

Freyheits-Concession und Begnadigung 72

Friedrichsfeld 26f., 31

Friedrichstadt 87 
Gallikanische Kirche/Église gallicane 142

Geheimer Rat zu Bayreuth 111f., 116

Geheimes Consilium, Geheimer Rat zu

Sachsen 52, 55, 61

Generalstaaten der Vereinigten

Provinzen/États-Généraux des

Provinces-Unies siehe/voir

Niederlande/Pays-Bas

Genf/Genève 4lf., 44, 62, 71f., 85f., 96$98,105,224,232,238$

Germersheim $25 f$.

Greifenstein 40

Grenoble 42

Griechenland/Grèce 185, 233

Groningen 224

Großziethen 159

Guyenne 7

\section{Hainaut 30}

Halberstadt 106, 202, 206

Halle 56, 62, 206, 235

Hamburg/Hambourg 11, 32, 41, 60, 125136, 142

Hanau 27, 33, 35-38, 43

Hanau-Lichtenberg 44

Heidelberg 17-31, 165

Heidelberger Katechismus 81

Heiliges Römisches Reich deutscher Nation/Saint Empire romain germanique $7-9,11,13,126$

Hessen/Hesse 33, 44, 48, 126, 130, 134, 160,231

Hessen-Darmstadt/Hesse-Darmstadt 44

Hessen-Homburg/Hesse-Hombourg 44

Hessen-Kassel/Hesse-Cassel 9, 11, 38, 43f., 71-83, 113, 116, 118

Hofgeismar 44

Hoheneck $119 f$.

Hohenlohe 44

Hohenzollern, -staat/État des 69, 79f., $139,142,145,150,193,212,216$, $235 f$.

Holland/Hollande siehe/voir

Niederlande/Pays-Bas

Homburg/Hombourg 35, 36

Impflingen 25

Irland/Irlande 8-10

Issoudun 44

Italien/Italie 231

ius reformandi 18,21
Juden/Juifs 35f., 125, 132f., 143, 145, 150,160

Kaiserslautern 18

Kalter Krieg/guerre froide 134

Karibische Inseln/Caraibes 133

Karlshafen, früher/jadis Sieburg 74

Kassel/Cassel 40, 44, 74

Kirchendisziplin/Discipline ecclésiastique $116,146,156,212,226$

Kleinziethen 159f., 206

Kleve/Clève 78, 142

Köln/Cologne 36, 142

Königsberg 205f., 214

Konsistorium/Consistoire der wallonischen und französischen reformierten Gemeinden in Deutschland bzw. der deutschen lutherischen Kirchen 28, 30, 32, 42, 52, 60f., 64, 76f., 92, 97, 101, 111, 115f., 150, 157166, 173, 176f., 196-203, 213, 222, 224

Köpenick 225

Kuba/Cuba 128

La Rochelle 130

Lambrecht 28-30

Languedoc 7,60, 159

Lausanne 40, 71-76, 232

Leiden/Leyde 19, 23, 28f., 99, 223, 229

Leipzig 32, 51, 55f., 60-70, 232, 234

Limburg/Limbourg 18

Lingen/Linange 92

Lingua franca 141,159

Lingua sacra 141

Lippe 44

Loire 7

London/Londres 238

Lorient 131

Lüttich/Liège 18, 27f., 30

Luzern/Lucerne 26

Lyon 41, 44, 234

Maastricht/Mastricht 33

Magdeburg/Magdebourg 30, 33, 62, 78, $100,106,156,205 f ., 232$

Main 35

Mainz/Mayence 35

Malmedy 18

Malta/Malte 41

Mannheim 17-33

Marburg/Marbourg 72,93

Meisenheim 29 
Meißen/Misnie 55-58

Metz 33, 159, 171

Mexiko/Mexique 134

Middelbourg, Seeland/Zélande 40

Moers/Mceurs 92

Mönchshofen/Mönchshofe 28

Montauban, Beschlüsse von/articles de 64

Montpellier 72

Morlaix 131

Mörlheim 26

Mülhausen/Mulhouse 99

Münchaurach 115

Nantes 128

siehe auch/voir aussi Edikt von/édit de

Nassau-Friesland/Nassau-Frise 85

Neckarschule 30

Neuchâtel 99, 238

Neudorf 27

Neuhaldensleben 206

Neustadt an der Aisch 107

Neustadt, Pfalz/Palatinat 18

Niederlande/Pays-Bas 8, 18, 22, 32, 40, 43f., 48, 55f., 68, 127f., 134, 212, 215$218,220,224,226,228,231 \mathrm{f}$

Niedersachen 9

Nordafrika/Afrique du Nord 151

Nordischer Krieg 55

Nördlingen 28

Nordsee/mer du Nord 127

Normandie 7, 130

Oberdirektorium/Grand Directoire, Conseil français für die französischen Kolonien in Brandenburg-Preußen 81

Oder 100

Offenbach 36

Oranien/Orange $11,80,85-106$

- Gerichtshof des Fürstentums/tribunal de 91

- Haus/Maison de 99, 101f., 106

Ordonnance française für die französischen Kolonien in Brandenburg-Preußen 77, 82

Oschatz 56-58

Osnabrücker Friedensvertrag (1648) 8

Ostsee/mer Baltique 127

Ottenhofen 122

Otterberg 18, 27f., 33
Paris 41, 159, 232-234, 238

Pépinière des Chantres et Maîtres d'École 194, 202-204, 208

Peru/Pérou 134

Pfalz/Palatinat 8f., 11, 17-34, 37, 40, 42, $46,48,80,107,160$

Pfälzischer Krieg/guerre de la Ligue d'Augsbourg 144

Picardie 40, 44

Piemont/Piémont 26, 44, 80

Poitou 7, 180

Portugal 126

Portugiesischer Unabhängigkeitskrieg/ guerre d'Indépendance du Portugal 133

Potsdam 156f.

siehe auch/voir aussi Edikt von/édit de

Potzlow 156, 232

Pouilly-sur-Loire 44

Pragela, Piemont 44

Prenzlau 156, 232

Raucourt 30

Regensburger Stillstand (1684) 7

République des lettres $12,159,228,231-$ 240

Revokation/Revocation siehe/voir Edikt von Fontainebleau

Rhein/Rhin 18, 22, 29, 32, 35, 86

Rheinsberg 236

Riga 130

Rijswijk/Ryswick, Friedensvertrag von/traité de 144

Rocroi, Schlacht von/bataille de (1643) 133

Rohrbach 25

Rom/Rome 185, 228, 233

Rotterdam 128

Rouen 131

Sachsen, Kur-/Saxe, Saxe électorale 11, 32, 51-70, 126, 130, 214

Saint-Sébastien 130

Saintonge 7

Sankt-Lambrecht, Kloster/Saint-

Lambrecht, couvent 18

Sanssouci 221

Santo Domingo/Saint-Domingue, île de 128,133

Saumur 31,72

Schlesien/Silésie 130f., 134, 205f., 236

Schönau/Schœnau 18, 27, 28, 30

Schweden/Suède 233 
Schwedt, Uckermark/Marche Ukraine $156,180,204$

Schweiz/Suisse $8,32,46,72,77,79,86$, 97f., 231, 235, 237

Seckenheim 26

Sedan 18, 27, 30f., 159

Siebenjähriger Krieg 65

Simon \& Arnail Fournier, Haus/maison 131

Skandinavien/Scandinavie 128

Solms-Braunfels 35, 44

Spanien/Espagne 126, 128, 131-135

Spree $100,226,228$

Stadelhof 115

Stavelot 18

Steinweiler 25

Stettin 234

Stockholm 130

Strasburg, Uckermark/Marche Ukraine 155-169, $205 f$.

Straßburg/Strasbourg 28, 38, 42, 232

Südafrika/Afrique du Sud 69

Tecklenburg/Tecklenbourg 92

Thiérache 27

Tirol/Tyrol 32

Torgau 55-59

Triest/Trieste 128
Uckermark/Marche Ukraine 33, 157, 159, 232

Utrecht 28,98

Vassy 37

Versailles 142

Verviers $18,23,28$

Vincennes 19

Vivarais 89

Wallonen/Wallons $17,29,31-33,38-40$, 140,150

Wallonie 36

Wallonische Kirche/Église wallonne 28, 31-33

Warschau/Varsovie 57

Wesel 36, 38, $205 f$.

Westfalen/Westphalie 18f., 131

Wien/Vienne 19

Wilhelmsdorf 108, 120-122

Windsheim $119 f$.

Wittenberg 60

Worms 28

Württemberg/Wurtemberg 9

Zweibrücken/Deux-Ponts 29, 43

Zweiter Weltkrieg/Seconde Guerre mondiale 180 